Internist 2011 · 52:5-6

DOI 10.1007/s00108-010-2783-2

Online publiziert: 24 . Dezember 2010

(c) Springer-Verlag 2010

\author{
M. Hallek ${ }^{1} \cdot$ R. Voltz ${ }^{3} \cdot$ H. Lehnert ${ }^{2}$. \\ ${ }^{1}$ Klinik I für Innere Medizin, Uniklinik Köln, Centrum für \\ Integrierte Onkologie (CIO) Köln/Bonn, Köln \\ ${ }^{2}$ Medizinische Klinik I, Universitätsklinikum Schleswig-Holstein, Campus Lübeck \\ ${ }^{3}$ Zentrum für Palliativmedizin, Uniklinik Köln, Centrum für \\ Integrierte Onkologie (CIO) Köln/Bonn, Köln
}

\title{
Was ist Palliativmedizin?
}

Angestoßen v. a. durch die politische Initiative, eine spezialisierte ambulante Palliativversorgung (SAPV) als Anspruch aller Versicherten in Deutschland gesetzlich zu verankern, ist der Begriff Palliativmedizin zurzeit in aller Munde. Dabei erscheint es oft so zu sein wie in der indischen Geschichte, in der eine Gruppe Blinder an einen Elefanten herangeführt und aufgefordert wird, diesen zu beschreiben: Der Eine, der einen Fuß berührt, beschreibt den Elefanten als rund und dick, der Zweite fasst den Schwanz an und beschreibt das Tier als lang und dünn, während der Dritte, den Stoßzahn anfassend, etwas „Kaltes“ und „Spitzes“ beschreibt. Alle haben sie Recht und doch beschreibt keiner den Elefanten in seiner Ganzheit. Was also ist Palliativmedizin?

Während es oft noch üblich ist, eine Erkrankung, sobald sie nicht mehr „kurativ“ zu behandeln ist, als „palliativ“ $\mathrm{zu}$ bezeichnen, haben sich durch die Entwicklung der spezialisierten Palliativmedizin in den letzten 27 Jahren in Deutschland die Begrifflichkeiten weiter differenziert. Demnach kann nach 3 übergeordneten Therapiezielen unterschieden werden: Heilung, Lebensverlängerung und Erhöhung der Lebensqualität.

Vor allem bei nicht mehr kurativ zu behandelnden Patienten ist die Reflektion dieser Therapieziele wesentlich. Dies betrifft erstens die kontinuierliche Evaluation des vorrangigen Therapieziels: Es gilt, den angemessenen Behandlungszeitpunkt zu identifizieren, ab dem eine Änderung des vorrangigen Therapieziels von Lebensverlängerung hin zur bestmöglichen Erhaltung der Lebensqualität sinnvoll ist. Zweitens sollte jede durchgeführte therapeutische oder diagnostische Maßnahme auf das von ihr zu erreichende vorrangige Therapieziel (Lebensverlängerung oder Lebensqualität) überprüft werden, um den Patienten im Rahmen des übergeordneten Therapieziels optimal zu behandeln.

Selbstverständlich können sekundäre Effekte auftreten: Insbesondere besteht die Hoffnung, dass die neu entwickelten Therapien („small molecules“ etc.), die zwar primär auf eine Verlängerung der Lebenszeit zielen, auch die Lebensqualität verbessern und Symptome lindern können. Auf der anderen Seite kann auch eine palliativmedizinische Behandlung sowie die Reduktion aggressiver Therapiemaßnahmen sekundär auch mit einer Verlängerung der Lebenszeit einhergehen. Dies hat vor kurzem die viel beachtete Publikation von Temel et al. [1] am Beispiel des nicht-kleinzelligen Bronchialkarzinoms (NSCLC) ergeben. In einer randomisierten Untersuchung (n: 151) wurden 2 Patientengruppen verglichen. Die Kontrollgruppe erhielt die routinenmäßige onkologische Versorgung, jedoch wurden die Kollegen ausdrücklich aufgefordert, bei Bedarf auch die Palliativmedizin zur Mitbehandlung hinzuzuziehen. Bei der Interventionsgruppe wurde zusätzlich zu einer definierten frühen Krankheitsphase (jeder Patient mit neu diagnostiziertem metastasiertem NSCLC) routinemäßig ein Palliativmediziner und eine Palliativpflegekraft mindestens einmal pro Monat in die Behandlung mit einbezogen („,early integration" - frühe Integration). Der Erstkontakt zum Patienten sollte zeitnah zur Erstdiagnose erfolgen. Patienten dieser Interventionsgruppe berichteten eine signifikant bessere Lebensqualität, zeigten signifikant weniger Symptome einer Depression, außerdem wurden signifikant weniger aggressive Therapiemaßnahmen durchgeführt. Darüber hinaus wurde eine Lebensverlängerung von 8 auf 11 Monate erzielt. Dies war zwar nur als sekundärer Endpunkt erhoben worden, unterstützt jedoch die oft beobachtete klinische Erfahrung.

\section{(7) Der Erstkontakt zum Patienten sollte zeitnah zur Erstdiagnose erfolgen}

Das bedeutet, dass sich die Therapieziele der Interventionen überschneiden können und dass daher die einzelnen therapeutischen und diagnostischen Maßnahmen im Einzelfall in Relation zu dem für den Patienten angemessenen und realistisch erreichbaren Therapieziel beurteilt werden sollten.

Um ein möglichst umfassendes Bild des „palliativen Elefanten“ zu beschreiben, diskutiert Prof. Müller-Busch, PastPresident der Deutschen Gesellschaft für Palliativmedizin, in diesem Heft zunächst einmal die unterschiedlichen Definitionen und Konzepte der Palliativmedizin. Danach berichten die Kölner Onkologen und Palliativmediziner von ihren Erfahrungen mit der Umsetzung der „frühen Integration" der Palliativmedizin in die onkologische Versorgung. Von vielleicht noch größerem Interesse für die Leserschaft wird der Artikel zu den palliativmedizinischen Aspekten nicht-onkolo- 
gischer Erkrankungen sein. Obwohl sich die spezialisierte Palliativmedizin international und national aus der Betreuung von Tumorpatienten heraus entwickelt hat und die Zusammenarbeit dort modellhaft für andere Bereiche sein kann, wird sich die Palliativmedizin bei NichtTumorpatienten in der Zukunft (der Gesetzgeber spricht von zukünftig $60 \% \mathrm{Tu}$ mor- und 40\% Nicht-Tumorerkrankten im Bereich der spezialisierten Palliativmedizin) deutlich an Bedeutung zunehmen. Gelten aber bei diesen Patienten die gleichen palliativmedizinischen Konzepte? Was sind vielleicht krankheitsspezifische Defizite, die verbessert werden müssen? Diesen Fragen gehen auch die darauf folgenden Arbeiten zur Symptomatologie (hier: Schmerz und Luftnot) und Komplementärtherapie nach.

Praktisch relevant für die Betroffenen ist, dass den Betroffenen in Deutschland 2011 entsprechende palliativmedizinische und hospizliche Hilfsangebote bisher noch nicht zuverlässig vom Gesundheitssystem angeboten werden. Hier müssen die Angehörigen selbst aktiv werden und bei den sich immer weiter verbreitenden Palliativ- und Hospiznetzen nachfragen, ob derartige Angebote für ihren kranken Angehörigen Sinn machen. Auf der anderen Seite können die primär behandelnden niedergelassenen Haus- und Fachärzte sowie Fachabteilungen der Krankenhäuser häufig noch keine Unterstützung durch palliativmedizinische Angebote erhalten, da der Aufbau einer qualifizierten und flächendeckenden palliativmedizinischen Infrastruktur noch nicht abgeschlossen ist.

Wissenschaftlich ist es zukünftig von großem Interesse, zukunftsorientierte Konzepte der Integration von klassisch internistischer Behandlung und palliativmedizinischem Denken zu formulieren und in ihrer Effizienz zu testen. Diese Konzepte müssen sich letztendlich an der Qualität der Versorgung messen lassen. Diese definiert sich z. B. durch die erzielte Lebenserwartung, der von den $\mathrm{Pa}$ tienten und ihren Angehörigen berichteten Qualität der Symptomkontrolle (Luftnot, Schmerz, Depression, Angst etc.), Lebensqualität und Autonomie (z. B. Sterben im häuslichen Umfeld, Möglichkeit Therapieentscheidungen gemeinsam auf der Basis der individuellen Patientenwünsche zu treffen) sowie der ausreichenden Beachtung psychosozialer und spiritueller Bedürfnisse.

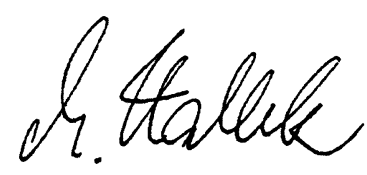

M. Hallek

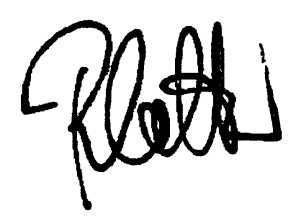

R. Voltz

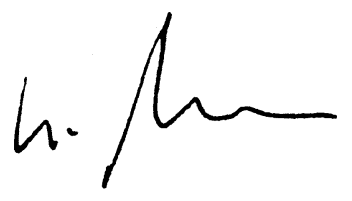

H. Lehnert

\section{Korrespondenzadresse}

Prof. Dr. R. Voltz

Zentrum für Palliativmedizin, Uniklinik Köln, Centrum für Integrierte Onkologie (CIO) Köln/Bonn

Kerpener Straße 62, 50937 Köln

raymond.voltz@uk-koeln.de

\section{Literatur}

1. Temel JS, Greer JA, Muzikansky A et al (2010) Early palliative care for patients with metastatic nonsmall-cell lung cancer. N Engl J Med 363: 733-742

\section{Patienten-Broschüre}

zum Thema

Erkältungskrankheiten

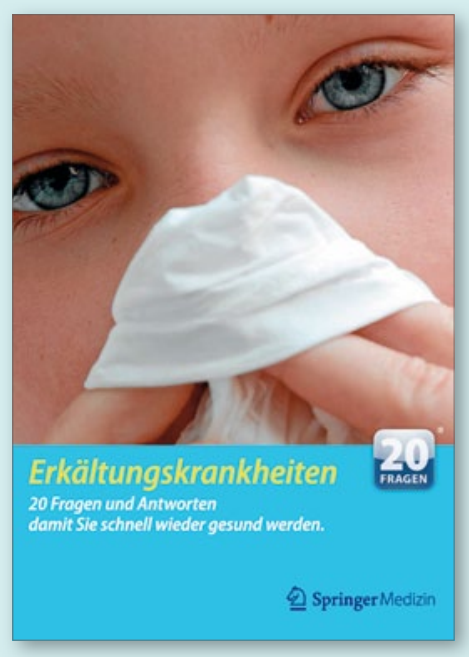

Winterzeit - Erkältungszeit: Viele Patienten haben jetzt ähnliche Fragen. Antworten auf die 20 häufigsten gibt eine kostenfreie Broschüre von SpringerMedizin. Dadurch werden Arzt und Praxisteam entlastet. Bei den Fragen geht es etwa um "Wieso bin ich schon wieder erkältet?" oder die oft gestellte Frage „Brauche ich ein Antibiotikum?". Die Antworten sind bewusst knapp gehalten, die Seiten aufgelockert gestaltet. Es werden erste Antworten auf die häufigsten Patientenfragen gegeben, keine umfassenden Abhandlungen. Was noch offen bleibt, kann der Patient zielgerichtet nachfragen - beim Praxisteam oder im Arztgespräch.

Die 32-seitige Broschüre im DIN A6-Format gibt es kostenfrei zur Auslage im Wartezimmer oder an der Theke. Es werden 100 Stück verschickt, wenn nicht mehr angegeben wird.

Bestellungen, per E-Mail an: vertrieb@ springer.com oder per Fax an: 06102/505-240 (Stichwort „Erkältungsbroschüre“. Wer mehr als 100 Broschüren wünscht, bitte angeben; Postanschrift nicht vergessen!) 